\title{
Primary or interval debulking surgery for stage IV ovarian cancer patients: experience from a ten-year case series
}

\section{Qiuling Deng}

Fudan University

\section{Zheng Feng}

Fudan University

\section{Hao Wen}

Fudan University

\section{Xingzhu Ju}

Fudan University

\section{Xiaojun Chen}

Fudan University

\section{Rui Bi}

Fudan University

Xiaohua Wu ( $\square$ docwuxh@163.com )

Fudan University

\section{Research}

Keywords: ovarian cancer, stage IV, primary debulking surgery, neoadjuvant chemotherapy, survival

Posted Date: June 3rd, 2020

DOI: https://doi.org/10.21203/rs.3.rs-32320/v1

License: (9) (i) This work is licensed under a Creative Commons Attribution 4.0 International License. Read Full License 


\section{Abstract \\ Background}

Whether patients with stage IV ovarian cancer can benefit from neoadjuvant chemotherapy (NACT) is unclear. The main aim was to compare the survival outcomes of primary debulking surgery (PDS) versus NACT followed by interval debulking surgery (IDS) in stage IV ovarian cancer patients.

\section{Methods}

This study retrospectively analyzed 190 consecutive patients with stage IV ovarian cancer who underwent either PDS or NACT + IDS between June 2003 and June 2013 at Fudan University Shanghai Cancer Center. Progression free survival (PFS) and overall survival (OS) were analyzed with Kaplan-Meier method. Multivariable cox proportional hazard models tested for associations of potential explanatory variables with survival.

\section{Results}

A total of 111 patients underwent PDS and 79 patients received NACT + IDS. More NACT patients had stage IVB disease compared with PDS patients $(p=0.001)$. Additionally, a higher rate of complete resection $(45.6 \%$ vs $21.6 \%, \mathrm{p}<0.001)$ was achieved in NACT patients compared to those with PDS. There was no significant difference of platinum sensitivity between the two groups $(p=0.998)$. The median PFS for PDS and NACT groups were $14(95 \% \mathrm{Cl}, 12-16)$ and $16(95 \% \mathrm{Cl}, 13-17)$ months, respectively $(\mathrm{p}=$ 0.744). The median OS were $40(95 \% \mathrm{Cl}, 31-49)$ and $53(95 \% \mathrm{Cl}, 41-65)$ months for PDS and NACT groups, respectively $(p=0.094)$. In addition, NACT was more beneficial than PDS in patients with extraabdominal lymph node metastases (median OS $(95 \% \mathrm{Cl})=37(24-50)$ vs $60(41-79)$ months, $p=0.006)$.

\section{Conclusions}

There were no significant survival differences between PDS and NACT groups for stage IV ovarian cancer patients. However, NACT had survival benefit in patients with extra-abdominal lymph node metastases.

\subsection{Background}

Ovarian cancer is one of the most lethal gynecological cancers worldwide [1]. Twelve to thirty-three percentage patients are diagnosed with stage IV disease, with an estimated 5-year survival of $20 \%$ [2]. The standard treatment includes debulking surgery and platinum-based chemotherapy. Patients who have no residual disease after debulking surgery achieve a better prognosis [3-6]. For poor surgical candidates or patients with a tumor burden that is too heavy to achieve complete resection, neoadjuvant chemotherapy (NACT) is a preferred alternative. 
However, whether patients can benefit from NACT remains controversial. Moreover, previous studies have always included stage III and IV patients together; thus, few studies have exclusively managed patients with stage IV disease to date. The post-hoc analyses of EORTC-55971 and CHORUS trials have suggested that patients with stage IV disease have significantly prolonged survival when they are in an NACT group compared with patients in a PDS group [7]. Other studies have tended to offer inconsistent conclusions [8-10].

Therefore, the primary aim of our study was to compare the survival outcomes of PDS and NACT followed by interval surgery in stage IV ovarian cancer patients. A secondary purpose was to discuss whether there was any survival benefit for specific subgroup with different FIGO stage IV subclassification and metastatic patterns.

\subsection{Methods}

\subsubsection{Patient selection and clinical data}

Patients with ovarian, fallopian tube, or primary peritoneal cancer who underwent either PDS or NACT followed by interval surgery between June 2003 and June 2013 at Fudan University Shanghai Cancer Center were identified from the hospital registry database. NACT was used in patients with bulking disease assessed by gynecologic oncologists and considered unlikely to achieve complete resection to $\mathrm{RO}$, or in patients who were poor surgical candidates. Two groups were formed according to the time of the first debulking surgery in the therapeutic sequence. For further evaluation, all patients were reclassified with respect to the revised FIGO (2014) system, and only FIGO stage IV patients were analyzed in our study. All clinical and imaging data of patients were re-reviewed by the experienced gynecologic oncologists and radiologists. The pathological diagnoses were reviewed, according to the WHO criteria, by two experienced gynecological pathologists.

\subsubsection{Definition}

Patients were subclassified according to their metastatic patterns: solely pleural effusion confirmed on cytology (stage IVA), parenchymal metastases (stage IVB), and extra-abdominal lymph node metastases (stage IVB). Patients with pleural effusion and positive extra-abdominal node metastases were categorized as extra-abdominal node metastases group. Patients with positive extra-abdominal node metastases and parenchymal metastases were categorized as parenchymal metastases group.

R0 was defined as the absence of macroscopic abdominal residual disease (RD) after surgery. All patients received platinum-based chemotherapy. Platinum sensitive was defined as a platinum-free survival of six months or longer, otherwise it was defined as platinum-resistant [11]. Progression-free survival (PFS) was defined as the time from the date of primary debulking surgery or the first cycle of NACT to the date of the first progression or recurrence [12]. Overall survival (OS) was defined as the time 
from the date of the primary debulking surgery or first cycle of NACT to the date of death or the last follow-up (September 20, 2019).

\subsubsection{Statistical analysis}

Patients were classified by treatment strategies into PDS and NACT groups. We compared categorical data using Chi-Square tests or Fisher's exact tests and continuous data using student's t tests or MannWhitney's U tests. PFS and OS were analyzed with Kaplan-Meier curves using log-rank tests in univariate analyses. The Cox proportional hazard model was used to estimate the Hazard Ratio (HR) and a 95\% confidence interval $(\mathrm{Cl})$ for multivariate analyses of prognosis. $\mathrm{P}<0.05$ was considered to be statistically significant, and all reported $P$ values were 2-sided. For these analyses, we used SPSS software, version 20.0 (IBM Inc, Armonk, NY, USA).

\subsection{Results}

\subsubsection{Patient characteristics}

A total of 190 patients with stage IV disease were included in our study, including 111 (58.4\%) patients who were placed in the PDS group and 79 (41.6\%) patients who were placed in the NACT group.

Table 1 summarizes selected patients' baseline characteristics. The median $₫ 33-82 \llbracket a g e$ at diagnosis was similar between the two groups ( 55 vs $54, p=0.283$ ). More patients were stage IVB compared with the PDS group $(78.5 \%$ vs $57.7 \%, p=0.001)$. Over $60 \%$ of patients in the NACT group had extra-abdominal lymph node metastasis, while pleural effusion constituted the largest proportion in the PDS group $(p<0.001)$. Histopathological type was significantly different between the groups $(p=0.003)$. A higher rate of complete resection had been achieved in NACT group compared to the PDS group (45.6\% vs $21.6 \%$, $p<0.001)$. The median (1-6) number of NACT cycles administered was two. Five of 79 patients were treated with neoadjuvant chemotherapy regimens other than platinum-based chemotherapy. All patients were administered platinum-taxane combination after debulking surgery. There was no significant difference of platinum sensitivity between the PDS and NACT groups $(35.1 \%$ vs $35.4 \%, p=0.998)$.

\subsubsection{Survival analysis}

The median follow-up was 35 months (range, $1-153)$. The median OS was 44 (95\% Cl, 35-53) months, with a 5 -year survival rate of $37 \%$. Figure 1 shows the Kaplan-Meier curves of PFS and OS stratified by the PDS and NACT groups. In univariate analyses, there were no significant differences of PFS (median $(95 \% \mathrm{Cl})=14(12-16)$ vs $15(13-17)$ months, $\mathrm{p}=0.744)$ and OS (median $(95 \% \mathrm{Cl})=40(31-49)$ vs $53(41-65)$ months, $p=0.094)$ between the two groups. Additionally, in patients with extra-abdominal lymph node metastases, NACT was associated with significantly better overall survival than PDS (median OS $(95 \% \mathrm{Cl})=37(24-50)$ vs $60(41-79)$ months, $p=0.006$; figure 2$)$, but not in patients with pleural 


\subsubsection{Potential explanatory variables with survival}

The known negative influences of the presence of residual disease ( $p=0.023$ and $<0.001$, respectively), and platinum resistance ( $p<0.001$ and $<0.001$, respectively) for PFS and OS were confirmed by univariate analyses. The same conclusion was reached in the multivariable models. However, there was no survival impact for FIGO stage IV sub-classification or metastatic patterns (Table 3 and Additional file 3).

\subsection{Discussion}

The comparison of PDS and NACT for advanced ovarian cancer has been under debate for several years; however, no consistent conclusion has been reached. Previous studies have always included stage III and IV together. Thus, few studies have exclusively managed patients with stage IV disease. Our study demonstrated that there were no survival differences between PDS and NACT groups for stage IV ovarian cancer patients. This finding is consistent with several previous studies. Seagle et al [9] observed that there was no difference between PDS and NACT among patients with stage IV disease in the American National Cancer Database with a median OS of 31.2 and 28.4 months, respectively. Chiva et al [10] also found no prognostic significance for patients with stage IV disease with NACT and PDS. However, other studies have demonstrated different opinions. The post-hoc analyses of EORTC-55971 and CHORUS trials have suggested that, although there was no survival benefit of NACT for the entire cohort, patients with stage IV disease had significantly prolonged survival in the NACT group compared with the PDS group [7]. Tajik et al [13] showed that stage IVB patients had a better OS and benefitted more from NACT compared with stage IVA patients, using the data of the EORTC-55971 trial. Whereas, criticize has been raised because the proportion of patient who were optimal cytoreduction was low and survival was inferior to that reported in aforementioned studies. Meyer et al [8] found that NACT was associated with impaired survival for stage IIIC compared with PDS, but not with stage IV disease (median OS: 31 vs 36 months) in the matched sample. The patients receiving chemotherapy alone were not included, since our study was retrospective. Thus, prospective studies are necessary in order to add reliable evidence to this difficult issue.

We found NACT was preferable in cases of extra-abdominal lymph node metastases. Unfortunately, there is no clear population for neoadjuvant chemotherapy, and few studies have been conducted. Considering that patients with FIGO stage IV are a highly heterogeneous group with possible survival differences in two treatment groups, more in-depth clinical and biological studies are needed to explore what specific population is suitable for neoadjuvant chemotherapy.

Additionally, sub-classifications of stage IV patients have been regarded as another important prognostic factor. Multiple studies reported similar survival results for stage IVA and IVB patients[14-16]. Several studies found a favorable prognosis of extra-abdominal lymph node metastases as the only site of 
distant disease[14, 17, 18]. While Jamieson et al [19] demonstrated no prognostic differences among different metastatic patterns. Our study indicated that neither sub-classification of stage IVA and IVB, nor three metastatic patterns impacted debulking surgical patients' outcomes. Our study excluded the patients never receiving surgery, surgical effort to no residual disease could blunt the effects of metastatic patterns and FIGO stage IV sub-classification. Also, Mert et al [20] did not support assignment to a higher FIGO stage (IV) based solely on depth of recto-sigmoid colon wall invasion. Our study included three patients with transmural bowel infiltration alone. Thus, we could not interpret whether the depth of bowel invasion was associated with survival.

Furthermore, some researchers have questioned whether NACT might induce chemoresistance. According to Luo et al [21] and Rauh-Hain et al [22], a significantly higher percentage of patients in the NACT group experienced platinum-resistant recurrences. Petrillo et al and Da Costa et al $[23,24]$ came to the same conclusion. Contrary to earlier reports, the proportion of platinum sensitivity was similar in our PDS and NACT groups. Regarding our study's retrospective nature, and the fact that partial platinum response information was lost, this should be interpreted with caution.

In addition, similar to previous publications, our study demonstrated that $\mathrm{R} 0$ resection was an independent prognostic factor for stage IV patients. The survival was similar between groups despite the fact that the NACT group had significantly greater RO resection in our study, this result might be due to NACT interfering with the perioperative visual estimation of tumor spread[25]. Thus, gynecological oncologists should make a maximal effort to control abdominal disease with surgery.

Limitations of this study include its retrospective inherence and potential selection bias, as we were unable to control, for instance, single institution bias. Moreover, patients receiving chemotherapy alone were not included. However, we followed up with our patients for up to 10 years, which could partially compensate for potential recall bias. Furthermore, we explored a homogeneous group of patients with stage IV ovarian cancer who underwent debulking surgery at the same institution with significant expertise in the treatment of ovarian cancer, which means that different surgery efforts may have been mitigated.

\subsection{Conclusions}

Our study found no significant survival differences between PDS and NACT groups for stage IV ovarian cancer patients. However, NACT had favorable survival in patients with extra-abdominal lymph node metastases.

\section{Declarations}

\section{Ethics approval and consent to participate}

This study was conducted according to the Declaration of Helsinki and approved by the Institutional review board (IRB) at the Fudan University Shanghai Cancer Center. As our study was non interventional 
and retrospective, formal written consent from patients included was not necessary.

\section{Consent for publication}

Not applicable.

\section{Availability of data and materials}

Not applicable.

\section{Competing interests}

The authors have no conflict of interest to declare.

\section{Funding}

No funding source to declare.

\section{Authors' contributions}

Concept and design: QD, ZF, HW. Data collection: QD, ZF. Statistical analysis: QD, ZF. Result interpretation: $\mathrm{QD}, \mathrm{ZF}, \mathrm{HW}, \mathrm{XW}$. Drafting and revising the manuscript: QD, ZF, HW, XJ, XC, XW. Pathology review: XJ, RB. All authors read and approved the final manuscript.

\section{Acknowledgements}

Not applicable.

\section{Tables}

Table 1. Baseline characteristics of the patients with PDS and NACT 


\begin{tabular}{|c|c|c|c|}
\hline \multirow[t]{2}{*}{ Characteristics } & PDS & NACT & \multirow[t]{2}{*}{$\mathrm{P}$ value } \\
\hline & $\mathrm{n}=111$ & $\mathrm{n}=79$ & \\
\hline \multirow[t]{2}{*}{ Age } & $55(33-82)$ & $54(36-75)$ & 0.283 \\
\hline & $78(70.3)$ & $59(74.7)$ & 0.504 \\
\hline$\geq 60$ & $33(29.7)$ & $20(25.3)$ & \\
\hline FIGO stage & & & 0.003 \\
\hline IVA & $47(42.3)$ & $17(21.5)$ & \\
\hline IVB & $64(57.7)$ & $62(78.5)$ & \\
\hline CA-125 level (U/mL) before cytoreduction surgery & $2650(7-39146)$ & $1098(9-5000)$ & 0.015 \\
\hline Histology & & & 0.003 \\
\hline High-grade serous & $98(88.3)$ & $56(70.9)$ & \\
\hline Poorly differentiated adenocarcinoma & $13(11.7)$ & $23(29.1)$ & \\
\hline Metastatic lesion & & & 0.001 \\
\hline Pleura & $47(42.3)$ & $17(21.5)$ & \\
\hline Extra-abdominal lymph nodes & $32(28.8)$ & $49(62.0)$ & \\
\hline Parenchymal and multiple organs & $32(28.8)$ & $13(16.5)$ & \\
\hline Residual disease $(\mathrm{cm})$ & & & 0.001 \\
\hline \multirow[t]{2}{*}{0} & $24(21.6)$ & $36(45.6)$ & \\
\hline & $87(78.4)$ & $41(51.9)$ & \\
\hline Unknown & $0(0)$ & $2(2.5)$ & \\
\hline Neoadjuvant chemotherapy cycles & - & $2(1-6)$ & \\
\hline$\leq 3$ & - & $66(83.5)$ & \\
\hline$>3$ & - & $13(16.5)$ & \\
\hline Platinum sensitivity & & & 0.998 \\
\hline Sensitive & $52(46.8)$ & $37(46.8)$ & \\
\hline Resistant & $39(35.1)$ & $28(35.4)$ & \\
\hline Unknown & $20(18.0)$ & $14(17.7)$ & \\
\hline
\end{tabular}

Table 2. Univariate analysis for PFS and OS. 


\begin{tabular}{|c|c|c|c|c|}
\hline \multirow[t]{2}{*}{ Variables } & \multicolumn{2}{|l|}{ PFS } & \multicolumn{2}{|l|}{ OS } \\
\hline & Months $\square 95 \% \mathrm{CI} \square$ & $\mathrm{P}$ value & Months $\square 95 \% \mathrm{CI} \square$ & $P$ value \\
\hline \multirow[t]{2}{*}{ Age } & & 0.245 & & 0.330 \\
\hline & $15(13-17)$ & & $46(33-59)$ & \\
\hline$\geq 60$ & $14(12-16)$ & & $38(27-49)$ & \\
\hline Treatment & & 0.744 & & 0.094 \\
\hline PDS & $14(12-16)$ & & $40(31-49)$ & \\
\hline NACT & $15(13-17)$ & & $53(41-65)$ & \\
\hline FIGO stage & & 0.929 & & 0.264 \\
\hline IVA & $14(12-16)$ & & $36(24-48)$ & \\
\hline IVB & $16(13-19)$ & & $47(37-56)$ & \\
\hline Metastatic lesion & & 0.633 & & 0.446 \\
\hline Pleura & $14(12-16)$ & & $36(24-48)$ & \\
\hline Extra-abdominal lymph nodes & $16(13-19)$ & & $48(34-62)$ & \\
\hline Parenchymal and multiple organs & $14(10-18)$ & & $44(22-65)$ & \\
\hline Residual disease (cm) & & 0.023 & & 0.001 \\
\hline \multirow[t]{2}{*}{0} & $18(11-25)$ & & $67(34-101)$ & \\
\hline & $14(13-15)$ & & $34(26-42)$ & \\
\hline Unknown & 13॰- & & $35(-)$ & \\
\hline Platinum sensitivity & & 0.001 & & 0.001 \\
\hline Sensitive & $23(19-27)$ & & $68(50-85)$ & \\
\hline Resistant & $10(9-11)$ & & $28(23-34)$ & \\
\hline Unknown & $13(-)$ & & $25(16-34)$ & \\
\hline
\end{tabular}

Table 3. Multivariate analysis for PFS and OS. 


\begin{tabular}{|c|c|c|c|c|}
\hline \multirow[t]{2}{*}{ Variables } & \multicolumn{2}{|l|}{ PFS } & \multicolumn{2}{|l|}{ OS } \\
\hline & HR (95\%CI) & $P$ value & HR (95\%CI) & $P$ value \\
\hline \multicolumn{5}{|l|}{ Age } \\
\hline & 1 & & 1 & \\
\hline$\geq 60$ & $1.394(0.931-2.088)$ & 0.107 & $1.156(0.799-1.737)$ & 0.408 \\
\hline \multicolumn{5}{|l|}{ Treatment } \\
\hline PDS & 1 & & 1 & \\
\hline NACT & $0.869(0.587-1.286)$ & 0.483 & $0.999(0.645-1.545)$ & 0.995 \\
\hline \multicolumn{5}{|l|}{ Metastatic lesion } \\
\hline Pleura & 1 & & 1 & \\
\hline Extra-abdominal lymph nodes & $1.215(0.783-1.883)$ & 0.385 & $1.125(0.705-1.795)$ & 0.621 \\
\hline Parenchymal and multiple organs & $1.221(0.758-1.967)$ & 0.412 & $0.878(0.539-1.430)$ & 0.602 \\
\hline \multicolumn{5}{|l|}{ Residual disease (cm) } \\
\hline \multirow[t]{2}{*}{0} & 1 & & 1 & \\
\hline & $1.547(1.023-2.341)$ & 0.039 & $2.466(1.536-3.960)$ & 0.001 \\
\hline Unknown & $4.507(1.039-19.556)$ & 0.044 & $2.696(0.620-11.719)$ & 0.186 \\
\hline \multicolumn{5}{|l|}{ Platinum sensitivity } \\
\hline Sensitive & 1 & & 1 & \\
\hline Resistant & $8.173(5.284-12.644)$ & 0.001 & $3.841(2.539-5.812)$ & 0.001 \\
\hline Unknown & $3.125(0.399-24.471)$ & 0.278 & $3.305(1.966-5.559)$ & 0.001 \\
\hline
\end{tabular}

\section{References}

[1] Bray F, Ferlay J, Soerjomataram I, Siegel RL, Torre LA, Jemal A. Global cancer statistics 2018: GLOBOCAN estimates of incidence and mortality worldwide for 36 cancers in 185 countries. CA Cancer $\mathrm{J}$ Clin. 2018;68(6): 394-424.

[2] Ataseven B, Chiva LM, Harter P, Gonzalez-Martin A, du Bois A. FIGO stage IV epithelial ovarian, fallopian tube and peritoneal cancer revisited. Gynecol Oncol. 2016;142(3): 597-607.

[3] Bristow RE, Tomacruz RS, Armstrong DK, Trimble EL, Montz FJ. Survival effect of maximal cytoreductive surgery for advanced ovarian carcinoma during the platinum era: a meta-analysis. J Clin Oncol. 2002;20(5): 1248-59.

[4] Chang S, Bristow RE, Ryu H. Impact of Complete Cytoreduction Leaving No Gross Residual Disease Associated with Radical Cytoreductive Surgical Procedures on Survival in Advanced Ovarian 
Cancer. Ann Surg Oncol. 2012;19(13): 4059-4067.

[5] Aletti GD, Dowdy SC, Gostout BS, Jones MB, Stanhope CR, Wilson TO, et al. Aggressive surgical effort and improved survival in advanced-stage ovarian cancer. Obstet Gynecol. 2006;107(1): 77

$-85$.

[6] Zivanovic O, Eisenhauer EL, Zhou Q, lasonos A, Sabbatini P, Sonoda Y, et al. The impact of bulky upper abdominal disease cephalad to the greater omentum on surgical outcome for stage IIIC epithelial ovarian, fallopian tube, and primary peritoneal cancer. Gynecol Oncol. 2008;108(2): 287-292.

[7] Vergote I, Coens C, Nankivell M, Kristensen GB, Parmar MKB, Ehlen T, et al. Neoadjuvant chemotherapy versus debulking surgery in advanced tubo-ovarian cancers: pooled analysis of individual patient data from the EORTC 55971 and CHORUS trials. The Lancet Oncology. 2018;19(12): 1680-1687.

[8] Meyer LA, Cronin AM, Sun CC, Bixel K, Bookman MA, Cristea MC, et al. Use and Effectiveness of Neoadjuvant Chemotherapy for Treatment of Ovarian Cancer. J Clin Oncol. 2016;34(32): 3854-3863.

[9] Seagle BL, Graves S, Strohl AE, Shahabi S. Survival After Primary Debulking Surgery Compared With Neoadjuvant Chemotherapy in Advanced Ovarian Cancer. Int J Gynecol Cancer. 2017;27(8): 16101618.

[10] Chiva L, Lapuente F, Castellanos T, Alonso S, Gonzalez-Martin A. What Should We Expect After a Complete Cytoreduction at the Time of Interval or Primary Debulking Surgery in Advanced Ovarian Cancer? Ann Surg Oncol. 2016;23(5): 1666-1673.

[11] Fung-Kee-Fung M, Oliver T, Elit L, Oza A, Hirte HW, Bryson P. Optimal chemotherapy treatment for women with recurrent ovarian cancer. Curr Oncol. 2007;14(5): 195-208.

[12] Rustin GJS, Vergote I, Eisenhauer E, Pujade-Lauraine E, Quinn M, Thigpen T, et al. Definitions for Response and Progression in Ovarian Cancer Clinical Trials Incorporating RECIST 1.1 and CA 125 Agreed by the Gynecological Cancer Intergroup (GCIG). Int J Gynecol Cancer. 2011;21(2): 419-423.

[13] Tajik P, van de Vrie R, Zafarmand MH, Coens C, Buist MR, Vergote I, et al. The FIGO Stage IVA Versus IVB of Ovarian Cancer: Prognostic Value and Predictive Value for Neoadjuvant Chemotherapy. Int J Gynecol Cancer. 2018;28(3): 453-458.

[14] Timmermans M, Sonke GS, Van de Vijver KK, Ottevanger PB, Nijman HW, van der Aa MA, et al. Localization of distant metastases defines prognosis and treatment efficacy in patients with FIGO stage IV ovarian cancer. Int J Gynecol Cancer. 2019;29(2): 392-397.

[15] Ataseven B, Harter P, Grimm C, Heitz F, Heikaus S, Traut A, et al. The revised 2014 FIGO staging system for epithelial ovarian cancer: Is a subclassification into FIGO stage IVA and IVB justified? Gynecol Oncol. 2016;142(2): 243-247. 
[16] Paik ES, Lee Y, Lee E, Choi CH, Kim T, Lee J, et al. Survival analysis of revised 2013 FIGO staging classification of epithelial ovarian cancer and comparison with previous FIGO staging classification. Obstetrics \& Gynecology Science. 2015;58(2): 124.

[17] Hjerpe E, Staf C, Dahm-Kahler P, Stalberg K, Bjurberg M, Holmberg E, et al. Lymph node metastases as only qualifier for stage IV serous ovarian cancer confers longer survival than other sites of distant disease - a Swedish Gynecologic Cancer Group (SweGCG) study. Acta Oncol. 2018;57(3): 331-337.

[18] Nasioudis D, Chapman-Davis E, Frey MK, Caputo TA, Witkin SS, Holcomb K. Should epithelial ovarian carcinoma metastatic to the inguinal lymph nodes be assigned stage IVB? Gynecol Oncol. 2017;147(1): 81-84.

[19] Jamieson A, Sykes P, Eva L, Bergzoll C, Simcock B. Subtypes of stage IV ovarian cancer; response to treatment and patterns of disease recurrence. Gynecol Oncol. 2017;146(2): 273-278.

[20] Mert I, Kumar A, Torres D, Huang Y, McGree ME, Weaver AL, et al. Should mucosal bowel invasion in ovarian cancer be assigned to FIGO stage IV disease? Gynecol Oncol. 2019;153(2): 238-241.

[21] Luo Y, Lee M, Kim HS, Chung HH, Song YS. Effect of neoadjuvant chemotherapy on platinum resistance in stage IIIC and IV epithelial ovarian cancer. Medicine. 2016;95(36): e4797.

[22] Rauh-Hain JA, Nitschmann CC, Worley MJ, Bradford LS, Berkowitz RS, Schorge JO, et al. Platinum resistance after neoadjuvant chemotherapy compared to primary surgery in patients with advanced epithelial ovarian carcinoma. Gynecol Oncol. 2013;129(1): 63-68.

[23] Da Costa AABA, Valadares CV, Baiocchi G, Mantoan H, Saito A, Sanches S, et al. Neoadjuvant Chemotherapy Followed by Interval Debulking Surgery and the Risk of Platinum Resistance in Epithelial Ovarian Cancer. Ann Surg Oncol. 2015;22(S3): 971-978.

[24] Petrillo M, Ferrandina G, Fagotti A, Vizzielli G, Margariti PA, Pedone AL, et al. Timing and Pattern of Recurrence in Ovarian Cancer Patients with High Tumor Dissemination Treated with Primary Debulking Surgery Versus Neoadjuvant Chemotherapy. Ann Surg Oncol. 2013;20(12): 3955-3960.

[25] Hynninen J, Lavonius M, Oksa S, Grénman S, Carpén O, Auranen A. Is perioperative visual estimation of intra-abdominal tumor spread reliable in ovarian cancer surgery after neoadjuvant chemotherapy? Gynecol Oncol. 2013;128(2): 229-232.

\section{Figures}




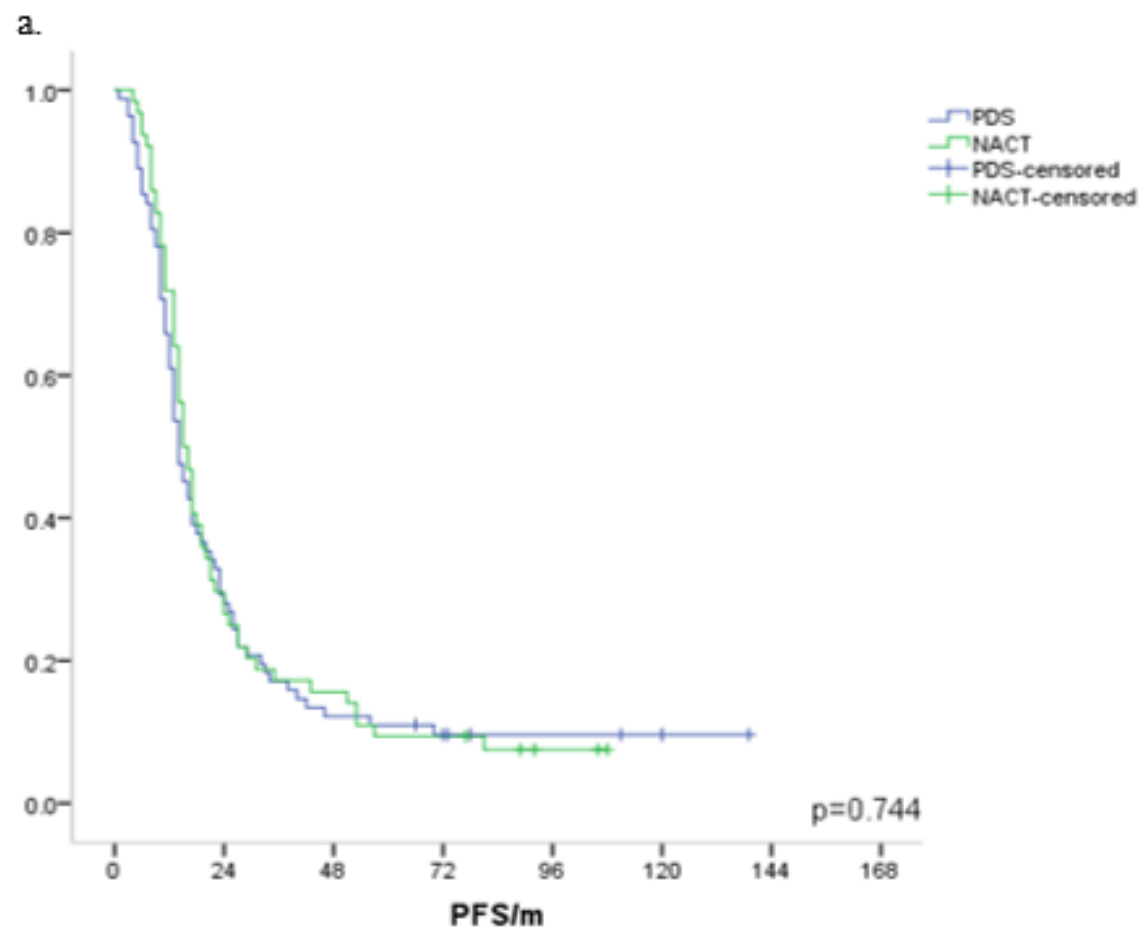

b.

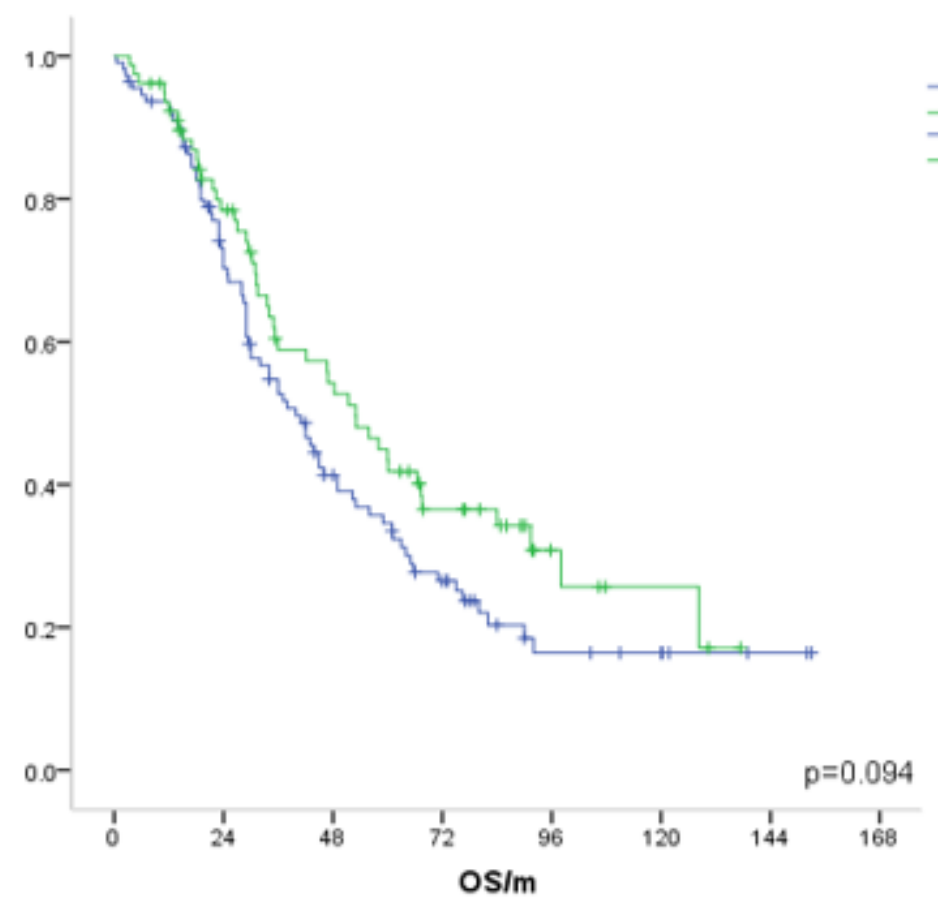

Figure 1

Kaplan-Meier survival curves of PFS and OS according to treatment. a. Kaplan-Meier survival curves of PFS according to treatment. b. Kaplan-Meier survival curves of OS according to treatment. 


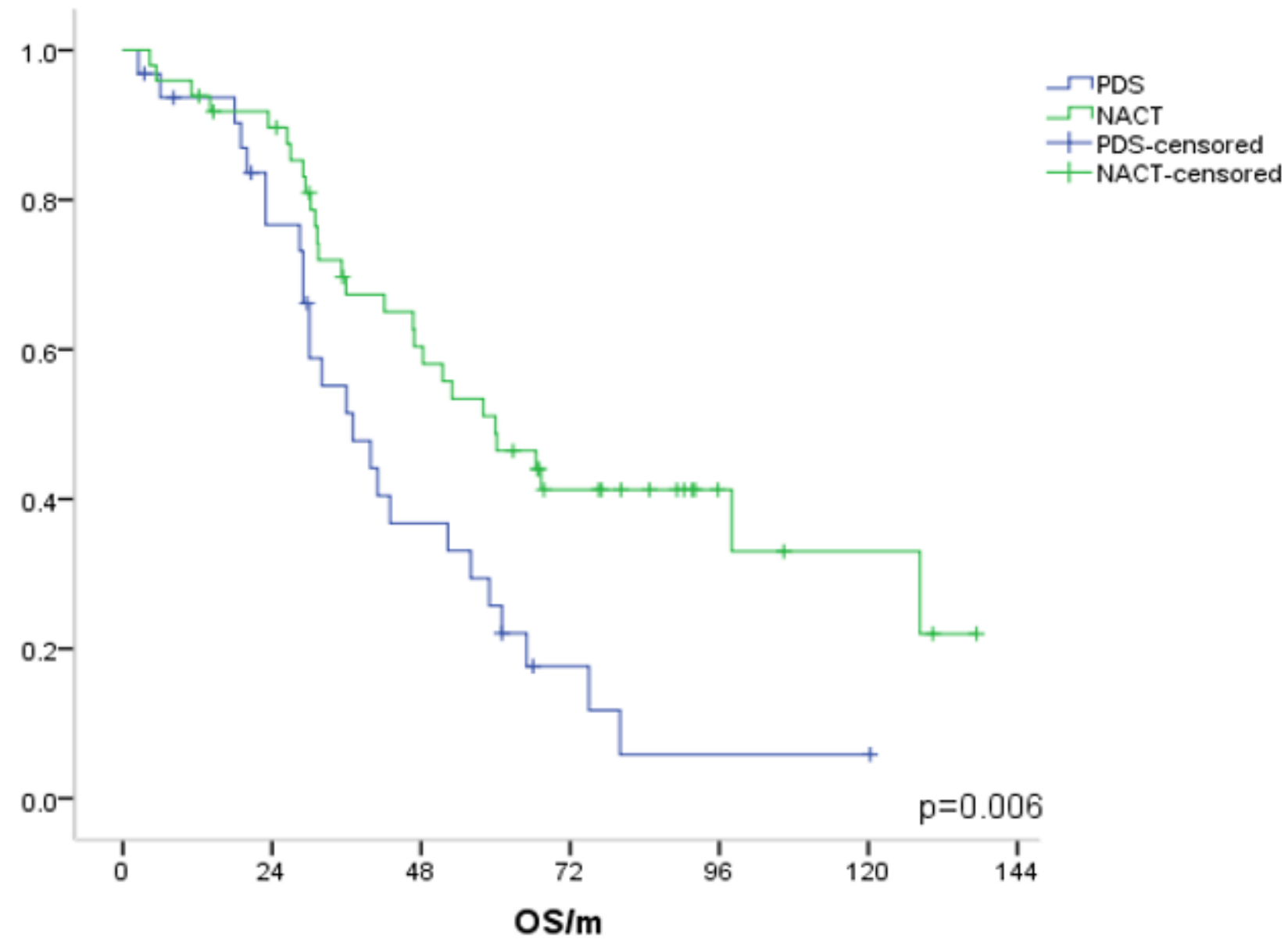

Figure 2

Kaplan-Meier survival curves of OS in patients with extra-abdominal lymph node metastases according to treatment.

\section{Supplementary Files}

This is a list of supplementary files associated with this preprint. Click to download.

- additionalfile1.docx

- additionalfile3.docx

- additionalfile2.docx 